\title{
Experiences, considerations and emotions relating to cardiogenetic evaluation in relatives of young sudden cardiac death victims
}

\author{
Christian van der Werf ${ }^{1}$, Astrid T Onderwater ${ }^{2}$, Irene $M$ van Langen ${ }^{3}$ and Ellen MA Smets ${ }^{\star, 2}$
}

Relatives of young sudden cardiac death (SCD) victims are at increased risk of carrying a potentially fatal inherited cardiac disease. Hence, it is recommended to perform an autopsy on the victim and to refer his or her relatives to a cardiogenetics clinic for a full evaluation to identify those at risk and allow preventive measures to be taken. However, at present, the number of families attending a cardiogenetics clinic after the SCD of a young relative is low in the Netherlands. We performed a qualitative study and report on the experiences and attitudes of first-degree relatives who attended a cardiogenetics clinic for evaluation. In total, we interviewed nine first-degree relatives and one spouse of seven SCD victims about their experiences, considerations and emotions before attendance and at the first stage of the cardiogenetic evaluation before DNA results were available. Interviews were transcribed verbatim and analysed. Medical professionals did not have an important role in informing or referring relatives to a cardiogenetics clinic. Importantly, all participants indicated that they would have appreciated a more directive approach from medical professionals, because their mourning process hampered their own search for information and decision-making. A need to understand the cause of death and wanting to prevent another SCD event occurring in the family were the most important reasons for attending a clinic. There are possibilities to improve the information process and better support their decision-making. The multidisciplinary cardiogenetic evaluation was appreciated, but could be improved by minor changes in the way it is implemented.

European Journal of Human Genetics (2014) 22, 192-196; doi:10.1038/ejhg.2013.126; published online 5 June 2013

Keywords: sudden cardiac death; cardiogenetics; experiences; considerations; emotions; qualitative research

\section{INTRODUCTION}

The sudden and unexpected loss of an apparently healthy young person, usually defined as a person under the age of 45 , is a major traumatic event for the bereaved. ${ }^{1}$ Inherited cardiac disease, in particular cardiomyopathies, primary arrhythmia syndromes and premature coronary artery disease, underlie at least half of such sudden cardiac death (SCD) cases. $^{2}$ Consequently, the relatives of young SCD victims are at increased risk of cardiovascular disease compared with the general population, possibly predisposing them to SCD. ${ }^{3}$ This is particularly true for young relatives.

To identify those relatives at risk of SCD, a thorough search for the cause of death of a SCD victim is crucial., ${ }^{2,4}$ Thus, a well performed autopsy, including storing DNA samples, is strongly recommended in these cases. ${ }^{5-7}$ If a possibly inherited cardiac disease is identified in the victim, the Heart Rhythm Society/European Heart Rhythm Association guidelines recommend that first-degree relatives be evaluated for the presence of that same condition. ${ }^{8}$ If no cause of death can be identified at the autopsy, or if no autopsy is performed, the guidelines also recommend cardiogenetic evaluation, ${ }^{8}$ because inherited cardiac diseases, particularly primary arrhythmia syndromes, are discovered in a third of the families. ${ }^{9}$

In the Netherlands, the relatives of a young SCD victim may be informed about the possible heritability of the cause of death and its potential implications by medical specialists if the victim is resuscitated or dies in hospital. In other cases, the general practitioner (GP) of the victim and/or the relatives has, in theory, a key role to inform and refer relatives. The GP may be aware of the recommendations for family evaluation after SCD or informed about them in the autopsy report.

So far, little is known about the true role of GPs in these situations or about the information and decision-making processes of relatives of young SCD victims in initiating an extensive trajectory of cardiogenetic evaluation. Because the issue of a cardiogenetic evaluation becomes relevant during the period of mourning, it may have a greater emotional impact than other forms of presymptomatic genetic evaluation. Understanding these issues in families is of particular significance, as only a small proportion of eligible relatives of young SCD victims actually attend a cardiogenetics clinic at present (unpublished data ${ }^{10}$ ).

Few studies have addressed the impact of SCD in the young on the bereaved. In one study, the parents of young SCD victims had a major need to understand the cause of death. ${ }^{1}$ In addition, many of these parents experienced a general lack of support from medical professionals. Others described the development of a system for investigating SCD in the young, including interviews with next-ofkin. ${ }^{11}$ As part of this procedure, the next-of-kin were informed about

${ }^{1}$ Department of Cardiology, Heart Failure Research Centre, Academic Medical Centre, Amsterdam, The Netherlands; ${ }^{2}$ Department of Medical Psychology, Academic Medical Centre/University of Amsterdam, Amsterdam, The Netherlands; ${ }^{3}$ Department of Genetics, University of Groningen, University Medical Centre Groningen, Groningen, The Netherlands

*Correspondence: Dr EMA Smets, Department of Medical Psychology, Academic Medical Centre/University of Amsterdam, P.0. Box 226601105 AZ, Amsterdam, The Netherlands. Tel: +31 20 5664768; Fax: +31 20 5669104; E-mail: e.m.smets@amc.uva.nl

Received 28 January 2013; revised 7 April 2013; accepted 10 May 2013; published online 5 June 2013 
their possible risks and the importance of screening. They much appreciated this information, but no follow-up of these relatives was conducted.

We conducted a qualitative interview study to gain insight into relatives' experiences, considerations and emotions during three distinct stages of the cardiogenetic evaluation process: stage (1), being informed about the link between sudden death in the young and inherited cardiac diseases and the recommendation to undergo evaluation; stage (2), the decision-making involved in following this recommendation and being evaluated; and stage (3), the initial cardiogenetic evaluation before definite results were available.

\section{METHODS}

\section{Setting and participants}

Our qualitative study was part of the CAREFUL (The yield of CARdiogenetic scrEening in First-degree relatives of sudden cardiac and UnexpLained death victims $<45$ years) initiative, which was designed to investigate the normal care provided after SCD in the young and to identify whether inherited cardiac disease was the cause of death. We also aimed to assess the efficacy of two interventions to improve this care. ${ }^{10}$

The present study was based on in-depth interviews with adult relatives of young victims of SCD. A qualitative approach was deemed most appropriate because we wanted to determine the possible impact of cardiogenetic evaluation in the relatives of young victims. Unlike quantitative research, aimed at testing a hypothesis in a large, randomly selected sample, qualitative inquiry focuses on small, selected samples to capture the most relevant variation in the study population. ${ }^{12}$ Participants were the first-degree relatives of SCD victims registered in the CAREFUL study as well as of other recently deceased young SCD victims who attended a cardiogenetics clinic for cardiological and genetic evaluation. The clinics were at the Academic Medical Centre, Amsterdam, or the University Medical Centre Groningen, Groningen, both in the Netherlands. During or after the first consultation with both a clinical geneticist or genetic counsellor and a cardiologist, information was collected on the SCD victim and the family. The strategy for further evaluation was then determined. This could include review of the victim's heart tissue if autopsy had been performed, retrieval of medical information on the victim and/or the relatives, cardiological evaluation of the relatives, and/or DNA testing in the victim or a relative. Support from a psychosocial worker was offered proactively.

The current study was introduced by the clinical geneticist or genetic counsellor to the relatives during or after the first consultation in order to include relatives at an early stage of the cardiogenetic evaluation trajectory. If they were interested, an information letter describing the study was sent to them and relatives were subsequently contacted by phone by the researchers to ask for their participation. In the Netherlands, it is common policy to exempt non-interventional qualitative research from ethical approval, as was the case in this study.

\section{Interviews}

After providing written informed consent, the participants were interviewed face-to-face by a physician ( $\mathrm{CvdW}$ ) or psychologist (AO). Interviews were conducted between April 2009 and February 2012. In order to ensure a safe environment for the participants, the interviews took place at their homes. Confidentiality was assured and the process of the interview was explained. The participants were invited to talk freely about their experiences and thoughts after the SCD of their young relative and the initiation of the cardiogenetic evaluation trajectory. The interviews were guided by open-ended main questions and a set of sub-questions. These were organized to cover their experiences, considerations and emotions during three stages of the trajectory: the information process (eg, 'Can you tell me how, after your beloved one died, you were informed about the possible heritability of the cause of death?'), the decision-making process (eg, 'What made you decide to visit the cardiogenetics clinic to initiate cardiac evaluation?') and the first phase of the cardiogenetic evaluation (eg, 'How did you experience the cardiological evaluation?'). Each interview lasted $45-75 \mathrm{~min}$ and was audio taped. After every interview, the interviewer summarized his or her impressions. All the interviews were transcribed verbatim.

\section{Data analysis}

Analysis was performed by one of the authors $(\mathrm{AO})$ following guidelines for qualitative research ${ }^{13}$ and using MAXQDA 2007 software (VERBI software, Marburg, Germany). Analysis was inductive, ie, no predefined theoretical frameworks were used, and it was aimed at identifying the most relevant themes. The analysis was started with open coding. Subsequently, two other authors (CvdW and ES) each coded one different interview independently. In a few meetings, the authors discussed the material and the codes to reach consensus. Subsequently, the text fragments were coded and sorted according to the identified themes and topics by one author $(\mathrm{AO})$. Gradually, open coding (summarizing and categorizing the data) was replaced with axial coding (confirmation of codes and the identification of broader relationships). ${ }^{14}$ Data were later clustered across interviews to derive some common themes. Constant comparison was used to explore emergent themes and to search for deviant cases. Two co-authors (CvdW and ES) critically reviewed the primary documents, coding schemes and interpretations as a quality check on the data.

\section{RESULTS}

Out of 22 eligible families, we were able to recruit nine first-degree relatives and one spouse representing seven families (including three interviews with two participants together). Table 1 shows the characteristics of our sample. The cardiogenetic evaluation trajectory was ongoing in all families and definite conclusions were not yet available.

To reduce the risk of individuals being identifiable, we have changed minor details in the quotations, which are used to validate and illustrate the meaning of each participant's comments. We removed verbal features from the quotations when this was deemed useful for clarity. The results are discussed according to the three predefined stages (the information process, decision-making process and the first phase of the cardiogenetic evaluation stage), followed by the role of the GP in the entire process. Results are described for these three stages separately.

\section{Information process}

Most participants reported that they had not been informed about the link between SCD in the young and inherited cardiac diseases by medical professionals. Some had searched the internet, whereas others were informed by relatives, friends or colleagues. Two participants reported that a relative or friend who was a physician had an important role in the information process:

\section{Table 1 Demographics of sudden cardiac death victims and participants}

\begin{tabular}{lllll}
\hline & \multicolumn{2}{c}{ Sudden cardiac death victim } & \multicolumn{2}{c}{ Participants } \\
Family no. & Gender & Age (years) & $\begin{array}{l}\text { Relationship } \\
\text { to victim }\end{array}$ & Age (years) \\
\hline 1 & Female & 40 & Sister & 43 \\
2 & Male & 39 & Father & 66 \\
3 & & & Mother & 65 \\
4 & Male & 35 & Sister & 33 \\
5 & Male & 41 & Sister & 49 \\
6 & Male & & Wife & 40 \\
7 & Male & 28 & Mother & 55 \\
& & & Father & 63 \\
& Male & 36 & Mother & 63 \\
\end{tabular}


The sudden death of my young brother was completely unexpected and a big shock. And then you start to think: my uncle also died at a young age, so maybe genetic factors could play a role? On the one hand, this horrible event was still in my head, but on the other hand, I also started to think about the impact for my children. (...) My uncle is an internist and he had heard about the high prevalence of a gene defect causing heart disease in a certain region of the Netherlands. He contacted the cardiogenetics clinic to make an appointment for us.

In only one case was the GP the first person to inform the victim's relatives about the indication for cardiogenetic evaluation. However, most participants said that neither their GP nor any other physician involved in the fatal event gave them this recommendation. All the participants indicated that they would have appreciated being informed about this recommendation by a medical professional, because it was not on their mind at first due to the mourning process. Importantly, one participant indicated that if she and her relatives had been informed about the possible role of genetic factors, they would have reconsidered their refusal to allow an autopsy on the victim.

\section{Decision-making process}

Most participants highlighted their disbelief and shock that someone young and healthy could pass away so suddenly. They consequently felt a strong desire to understand the cause of the victim's death:

To me, the most important is: 'Why? What on earth happened?' That boy was so healthy, never had anything, was always playing sports.

Several participants thought knowing the cause of death would give them some peace of mind. This seemed to be part of the mourning process, and they were therefore keen to learn about a possible genetic predisposition.

Participants talked about the fear of dying suddenly. The possibility that they or their relatives could pass away suddenly was termed 'impossible to live with'. Several participants tried to set their continuous fear aside by realizing that other relatives had reached old age in a healthy state.

However, the most important reason to initiate cardiogenetic evaluation was to prevent a second fatal event in the family, especially in the participants' children or grandchildren:

My brother-in-law did say once: 'Do you really want to know everything? What if you know and there is nothing you can do to change things?' Then I started to have doubts, but eventually I thought: we want to grow old, but our grandson is more important. If he should have something that could be controlled by medicine or something else, well, that's more important to us than our own health.

Some participants felt that they had no choice but to be evaluated: sticking their heads in the sand was not an option. In addition, several already had possible cardiac symptoms, which frightened them but was even more the reason to be evaluated.

Feelings of guilt of passing on the genetic susceptibility to SCD were expressed by some of the participants who had lost a child or grandchild:

My mother found it very difficult to know that she had something wrong in her DNA. She felt guilty. We kept telling her that she couldn't influence which parts of DNA that were passed on or not.

In addition, one participant felt guilty about her own action during the event, because when her son had phoned her because he was having palpitations, she had reassured him shortly before his death. However, she reported that these feelings of guilt had diminished after discussing the event and her actions with medical professionals. Similar feelings of guilt were reported by relatives who were present during the event and who had tried to resuscitate the victim.

When characterizing their own and other relatives' coping behaviour, participants often used the word 'rational'. However, we noticed this term was used in two ways. Some meant that any available test to identify the cause of death and its possible implications should obviously be taken. In contrast, others meant that there was no use searching for the cause of death, because it would not bring their relative back.

Some drawbacks of cardiogenetic evaluation were also specified by participants, for example, bringing back painful memories and the possible consequences for insurance policies and mortgages. Participants also reported that some doubts remained or arose during the process:

What if something is identified and no effective therapy is available?

Perhaps it's better not to know everything.

Isn't all this extensive cardiogenetic evaluation rather over-the-top?

The majority of participants started the cardiogenetic evaluation directly or within a few months of the victim's death. However, some admitted to having allowed themselves more time to consider the evaluation, because it hurt too much to think about it shortly after the fatal event.

Deciding to attend a cardiogenetics department gave a feeling of serenity to several participants. Those who postponed the decision to be evaluated sometimes regretted afterwards that they were not evaluated earlier, because this would have given them more clarity at an earlier stage. In addition, one participant was angry because none of the relatives or other individuals involved had persuaded him to have an autopsy performed on the SCD victim or to have himself evaluated:

Afterwards I was kind of angry. Why did none of my relatives say: you need to have what happened checked out? (...) We didn't want to, because we didn't want someone to cut into him. (...) We were too emotionally involved. Someone who was a little less involved could have said something, but nobody did.

\section{Cardiogenetic evaluation}

When the participants decided to attend a cardiogenetics clinic, some were pleasantly surprised by how quickly an appointment was made, whereas others were discontent about having to wait for several weeks.

The majority of participants were positive about the first appointment at the clinic. The counsellor's attitude was experienced as warm and supportive. In addition, the verbal and written information given was appreciated. Nevertheless, several participants were disappointed by the fact that the cardiological examination was not performed directly. Instead, they were referred to a cardiologist which, in their opinion, caused unnecessary delay for the entire trajectory. In general, 
participants noted that a faster cardiogenetic evaluation would be needed to bring them peace of mind in their state of fear and insecurity.

At the genetic counselling of families with a possible inherited cardiac disease, the attending individuals are usually advised to inform their other relatives. Thus, the ones first attending a counselling have a central role in the informed decision making for other relatives. Most participants took on the task to inform their relatives about a possible genetic cause of death and the importance of cardiogenetic evaluation. One participant explained that it was hard to inform relatives about something that was difficult for him as well. Most relatives wanted to participate in the cardiogenetic evaluation. However, there were also reports of relatives who made critical comments, refused to participate, or who did not want to be informed about the results. One participant was very grateful to a relative who motivated the others to be evaluated:

I have some medical knowledge, but I would never have initiated this evaluation. He's gone, just a heart attack, dead is dead. But you [the relative who initiated the cardiogenetic evaluation] had very different thoughts. Looking back, I think you did very well to search the internet and find relevant information. (...) Your actions have been very important to me, to the children, and to the entire family.

During the first appointment in the clinic, most participants felt well cared for, although there were differences between the physicians and counsellors that they met. In general, the fact that someone was looking into a possible inherited disease in their family was experienced as an act of support. In addition, they felt that their grief was being understood, although most participants did not feel the need for the psychological support that was also offered.

\section{Role of the GP}

Some participants felt that, during the evaluation, they were mainly being supported by their close circle of relatives and friends. One participant highlighted that it was easier to discuss genetics with someone who is not a direct relative. Most participants expected their GP to have a supportive role, but had varying experiences with the support he or she provided. Some GPs were proactive by explaining the autopsy report and, in one case, advising cardiogenetic evaluation. Other GPs supported the participants by stopping by, giving advice and listening. Some GPs were not supportive; they doubted the value of cardiogenetic evaluation and refused to refer the participants because of insufficient information about the SCD case. Some GPs were not involved at all; they did not contact the participants after the SCD nor did the participants contact their GP.

\section{DISCUSSION}

By exploring relatives' experiences, considerations and emotions relating to cardiogenetic evaluation following the sudden death of a young relative, we can tentatively conclude that medical professionals had not played an important role in informing and referring the participants in this study to a cardiogenetics clinic. Importantly, all the participants indicated that they would have appreciated being informed about this recommendation by a medical professional, because it was not on their mind at first due to the mourning process. These are essential findings, given the current discrepancy between the recommendation for cardiogenetic evaluation of relatives of young $\mathrm{SCD}$ victims and the small number of families that currently visit a cardiogenetics clinic.
Our data may suggest that the GP or other medical professionals had not had a key role in the information and referral process. Most participants, ie, the ones that attended the cardiogenetics department, demonstrated an active coping style, including performing their own research on the internet, sometimes initiated or supported by significant others, and by self-referral. Although these data may be influenced by selection bias, we find it worrying information, because it might indicate that the current information process in the case of young SCD is poorly organized. In a focus group study among GPs and coroners, a lack of knowledge about cardiogenetics (eg, the link between sudden death in the young and inherited cardiac diseases) was identified as one of the reasons for the low referral rate of families for cardiogenetic evaluation (unpublished data). GPs and other medical professionals involved in an SCD may also be reluctant to burden mourning relatives with information on a possible genetic predisposition. The introduction of case managers to guide the medical and judicial issues in SCD of young individuals and their relatives was one of the study's recommendations. In addition, the study recommended that a contact person should follow-up families when an SCD victim dies in hospital, as part of the bereavement care, ${ }^{1}$ so advice on cardiogenetic evaluation could be incorporated in this.

Research into the perceptions and mourning reactions of relatives confronted with an SCD show they have a great need to understand the cause of death. ${ }^{1,15}$ Indeed, this was an important reason for undergoing cardiogenetic evaluation among our participants. Participants were also motivated by reducing the chance of another SCD event in their family.

In cardiogenetics, the psychological impact of presymptomatic genetic evaluation has been studied in patients with the most prevalent inherited cardiac diseases: the congenital long QT syndrome (LQTS) and hypertrophic cardiomyopathy (HCM). In a longitudinal study by our centre, addressing the psychological consequences of predictive genetic testing for LQTS, the short-term increase in distress levels returned to normal after 18 months. ${ }^{16}$ However, distress levels were high at the first consultation and after 18 months in individuals with an uncertain electrocardiogram in whom a pathogenic mutation was identified. In another study, parents of carrier children at risk for symptoms of LQTS were also preoccupied with the disease for at least 18 months. ${ }^{17}$ This is most probably caused by a combination of uncertainty of being affected during the first consultation and by the consequences of being affected in the long term.

Our group also reported on the quality of life and psychological distress in $228 \mathrm{HCM}$ mutation carriers. ${ }^{18}$ While the overall outcomes were similar to the general Dutch population, the quality of life was significantly better in mutation carriers without phenotypic signs of HCM who were tested predictively.

Altogether, presymptomatic genetic evaluation seems to cause some psychological distress at the beginning of the trajectory, possibly due to the uncertainty about who might be affected and at risk for SCD. However, cardiological evaluations showing the absence of manifest disease or disease in an initial phase might also provide some reassurance. In the longer term, the level of psychological distress in adults seems to be similar or even less than in the general population, whereas the level of distress may remain high in the parents of affected children. Genetic counselling, cardiological evaluation and prophylactic measures may take away some of the uncertainties.

To the best of our knowledge, this is the first study to report on the psychological impact of presymptomatic cardiogenetic evaluation in the relatives of young SCD victims. This population differs from individuals who undergo predictive testing for LQTS or HCM in various aspects. First, this population probably suffers from more 
insecurity, because instead of being tested for the presence or absence of LQTS or HCM, the outcome of cardiogenetic evaluation of the relatives may be more variable. An inherited cardiac disease may be diagnosed, not diagnosed but still considered likely (requiring additional testing or close follow-up), or entirely excluded. Second, in the relatives of young SCD victims, the cardiogenetic evaluation is initiated during the period of mourning, which may well aggravate its emotional impact. Indeed, participants reported that they did not think about cardiogenetic evaluation or postponed their decision to undergo evaluation because they were too emotional in the period following the SCD. Yet they would have appreciated being informed about their own possible risk. This is in agreement with findings described by Mukerji et al, ${ }^{11}$ who interviewed relatives of young SCD victims in Michigan. They described how none of the relatives expressed concern about being contacted for extra information on the SCD victim or being informed about the possible risk and the importance of screening.

At the cardiogenetics clinics, the approach to a family with SCD is multidisciplinary. ${ }^{19,20}$ One major goal is to provide ongoing support to these families, partly through the constant availability of accurate and up-to-date information on the evaluation process by the clinical geneticist or genetic counsellor. In addition, in our clinics, psychological support is mandatory when children are directly involved, and it is also offered to all other families. The need for support and the feelings of fear and guilt that were expressed in this study underscores the value of this multidisciplinary approach in such a clinic.

In the interpretation of our findings, several considerations should be taken into account. First, we recognize the impact of the selfselection of participants on our findings. They may demonstrate specific perceptions and experiences of cardiogenetic evaluation, especially because most participants had an active coping style. We cannot assume that our results would apply to all members of these families, in particular because those relatives who refused to be evaluated were not interviewed.

Second, the data was based on a Dutch sample, whose experiences may not apply to other populations, in particular in the light of specific Dutch regulations. For example, in other countries, autopsy is required by law for an unexplained death and medical professionals other than the GP may have a central role in informing and referring relatives for cardiogenetic evaluation. Thus, it is unknown whether our results also apply to countries in which the health care in young SCD cases is organized differently.

Third, our numbers were small because few relatives of SCD cases registered in the CAREFUL study actually attended our cardiogenetics clinics during the study period. In contrast to quantitative research, a small sample size is of less concern in this type of qualitative research. ${ }^{21}$ Qualitative studies aim to provide insight into complex psychosocial issues and understanding thereof. An appropriate sample size for a qualitative study usually becomes obvious as the study progresses and new themes or explanations stop emerging from the data (ie, data saturation). Indeed, we observed a significant overlap in the experiences and attitudes of participants. Altogether, we believe that our methods generated rich data.

In conclusion, our findings imply that, in the Netherlands, the trajectory between the SCD of a young individual and the cardiogenetic evaluation of the victim's relatives is complex, both logistically and psychologically. The trajectory includes the information and decision-making processes. There are possibilities to improve the information process and support the decision-making process, for example, by introducing case managers. The multidisciplinary cardiogenetic evaluation was appreciated by our participants and this could be further improved by minor organizational changes, such as starting the cardiological examination during the first visit to the cardiogenetics clinic.

\section{CONFLICT OF INTEREST}

The authors declare no conflict of interest.

\section{ACKNOWLEDGEMENTS}

This study was funded by ZorgOnderzoek Nederland Medische Wetenschappen (ZonMW, grant number 120610013). We thank the participants and the cardiogenetics clinics of the Academic Medical Centre, Amsterdam, and the University Medical Centre Groningen, Groningen for help in recruiting the participants. We also thank Jackie Senior for editing the final version of the text.

1 Wisten A, Zingmark K: Supportive needs of parents confronted with sudden cardiac death-a qualitative study. Resuscitation 2007; 74: 68-74.

2 van der Werf C, van Langen IM, Wilde AA: Sudden death in the young: what do we know about it and how to prevent? Circ Arrhythm Electrophysiol 2010; 3: 96-104.

3 Ranthe MF, Winkel BG. Andersen EW et al: Risk of cardiovascular disease in family members of young sudden cardiac death victims. Eur Heart J 2013; 34: 503-511.

4 Skinner JR, Duflou JA, Semsarian C: Reducing sudden death in young people in Australia and New Zealand: the TRAGADY initiative. Med J Aust 2008; 189: 539-540

5 Basso C, Burke M, Fornes $P$ et al: Guidelines for autopsy investigation of sudden cardiac death. Virchows Arch 2008; 452: 11-18.

6 Semsarian C, Hamilton RM: Key role of the molecular autopsy in sudden unexpected death. Heart Rhythm 2012; 9: 145-150.

7 Ferrero-Miliani L, Holst AG, Pehrson S, Morling N, Bundgaard H: Strategy for clinical evaluation and screening of sudden cardiac death relatives. Fundam Clin Pharmacol 2010; 24: 619-635

8 Ackerman MJ, Priori SG, Willems S et al: HRS/EHRA expert consensus statement on the state of genetic testing for the channelopathies and cardiomyopathies: this document was developed as a partnership between the Heart Rhythm Society (HRS) and the European Heart Rhythm Association (EHRA). Europace 2011; 13 1077-1109.

9 van der Werf C, Hofman N, Tan HL et al: Diagnostic yield in sudden unexplained death and aborted cardiac arrest in the young: the experience of a tertiary referral center in The Netherlands. Heart Rhythm 2010; 7: 1383-1389.

10 Hendrix A, van der Werf C, Bots ML et al: Rationale and design of the CAREFUL study: The yield of CARdiogenetic scrEening in first degree relatives of sudden cardiac and unexpLained death victims <45 years. Neth Heart J 2010; 18: 286-290.

11 Mukerji S, Hanna B, Duquette D, Bach J, Rosenman K: Sudden cardiac death of the young in Michigan: development and implementation of a novel mortality review system. J Community Health 2010; 35: 689-697.

12 Bernhardt BA: The role of qualitative research in medical genetics: listening to the voices of our patients. Am J Med Genet A 2008; 146A: 3132-3135.

13 Plochg T, van Zwieten M: (eds) Guidelines for Quality Assurance in Health and Health Care Research: Qualitative Research. Amsterdam: Qualitative Research Network AMC-UvA, 2002.

14 Murray M, Chamberlain K: (eds) Qualitative Health Psychology: Theories and Methods, London: SAGE, 1999

15 Merlevede E, Spooren D, Henderick $\mathrm{H}$ et al: Perceptions, needs and mourning reactions of bereaved relatives confronted with a sudden unexpected death. Resuscitation 2004; 61: 341-348.

16 Hendriks KS, Hendriks MM, Birnie E et al: Familial disease with a risk of sudden death: a longitudinal study of the psychological consequences of predictive testing for long QT syndrome. Heart Rhythm 2008; 5: 719-724.

17 Hendriks KS, Grosfeld FJ, van Tintelen JP et al: Can parents adjust to the idea that their child is at risk for a sudden death?: psychological impact of risk for long QT syndrome. Am J Med Genet A 2005; 138A: 107-112.

18 Christiaans I, van Langen IM, Birnie E, Bonsel GJ, Wilde AA, Smets EM: Quality of life and psychological distress in hypertrophic cardiomyopathy mutation carriers: a crosssectional cohort study. Am J Med Genet A 2009; 149A: 602-612.

19 Ingles J, Semsarian C: Sudden cardiac death in the young: a clinical genetic approach. Intern Med J 2007; 37: 32-37.

20 van Langen IM, Hofman N, Tan HL, Wilde AA: Family and population strategies for screening and counselling of inherited cardiac arrhythmias. Ann Med 2004, 36:Suppl 1 116-124.

21 Marshall MN: Sampling for qualitative research. Fam Pract 1996; 13: 522-525. 